\title{
SWITCHED-DIVERSITY TRELLIS-CODED 8-DPSK FOR MOBILE RADIO APPLICATIONS
}

\author{
G. FEMENIAS and R. AGUSTI
}

Dept. de Teoria del Senyal i Comunicacions

Universitat Politècnica de Catalunya (UPC)

Apdo. 30.002, 08080 Barcelona - Spain

\section{ABSTRACT}

In this paper the performance of $64 \mathrm{Kbits} / \mathrm{s}$ two-branch switched-diversity 4DPSK and TCM-8DPSK systems in the presence of a simulated Rayleigh fading channel is analized. BER is obtained as a function of switching threshold, maximum doppler shift frequency of the channel and interleaving buffer size. In particular, the optimum switching threshold is determined as a function of the other parameters. It is found that its location is nearly constant for different interleaving buffer sizes and practically unaffected by the doppler frequency. Using the optimum threshold the BER-performance of the systems is determined with doppler frequency and interleaving buffer size as parameters. One of the most significant results is that the switcheddiversity TCM-8DPSK system improves the switcheddiversity 4DPSK performance in more than $8 \mathrm{~dB}$ for a BER $\leq 10^{-3}$, a doppler frequency of $5 \mathrm{~Hz}$ and an interleaving buffer size of 20x5000 symbols. By decreasing this figure to $10 \times 1000$ the improvement is only reduced by $1.5 \mathrm{~dB}$.

\section{INTRODUCTION}

The performance of a high capacity mobile radio system operating in the UHF band is severely affected by the multipath propagation medium. The presence of deep fades, characteristic of the Rayleigh fading environment, produces high error rates, even with high average signal to noise ratios. To counteract the effects of the fading one can resort to coding, diversity or combined coding/diversity techniques.

The advantages of using diversity techniques have been largely invoked for mitigating the effects of a Rayleigh fading channel. This technique, when used at the receiver, uses independent faded signals received on more than one physically separated antenna. In particular, space diversity has been envisaged in UHF mobile communications because both experimental and theoretical results indicate that it can be achieved with very small antenna spacings, usually of the order of one wavelength or less [1]. The space diversity, however, has problems derived from the increasing complexity of the receiver when signal combining methods such as equal-gain or even selection are to be used. These diversity techniques require continuous know- ledge of all the received signals and, when implemented at the receiver, a separate receiver chain is needed for each signal to be combined or selected, which adds to the receiver complexity. A simpler diversity technique is switched diversity and, among all of the switching strategies, one of the most interesting is the so-called "switch and stay strategy" [2], because it avoids excessive switchings when both signals are simultaneously faded. With this strategy, the instantaneous power of the received signal is monitored. The single receiver input remains connected to one of the diversity antennas until the signal fades below a given threshold. When the instantaneous power crosses the threshold in the negative direction, the receiver input is switched to another antenna in the hope that, due to the decorrelation between the received amplitudes, the new received signal level will be above the threshold. One of the problems that appears when dealing with switched diversity systems is the high error probability of the symbols received during the transient following the switch.

Trellis Coded Modulation (TCM), as introduced by Ungerboeck [3], has recently become a popular means of achieving coding gain on the AWGN channel [4] and also on the Rayleigh fading channel [5], [6]. Furthermore, the gain is available without extra bandwidth requirements and with simple decoders utilizing the Viterbi algorithm. Our aim in this paper is to investigate to what extent the switching errors, in addition to those due to thermal noise, can be reduced using a proper TCM scheme suitable for mobile radio environments. In particular, in this paper we assess the performance of the "switch and stay" diversity TCM-8DPSK modulation scheme with 8 state Ungerboeck code, that is, a combined coding/diversity technique. Also, we consider only the case where interleaving/deinterleaving is employed to further combat the Rayleigh fading.

\section{SYSTEM MODEL}

The equivalent baseband transmission system is shown in Fig. 1. We have assumed the overal transfer function to be, in absence of channel distortion, a raised-cosine with a 0.5 roll-off parameter equally splitted into transmitter and receiver. In order to emphasize only the effects of the coding/diversity set-up, perfect automatic fre- 


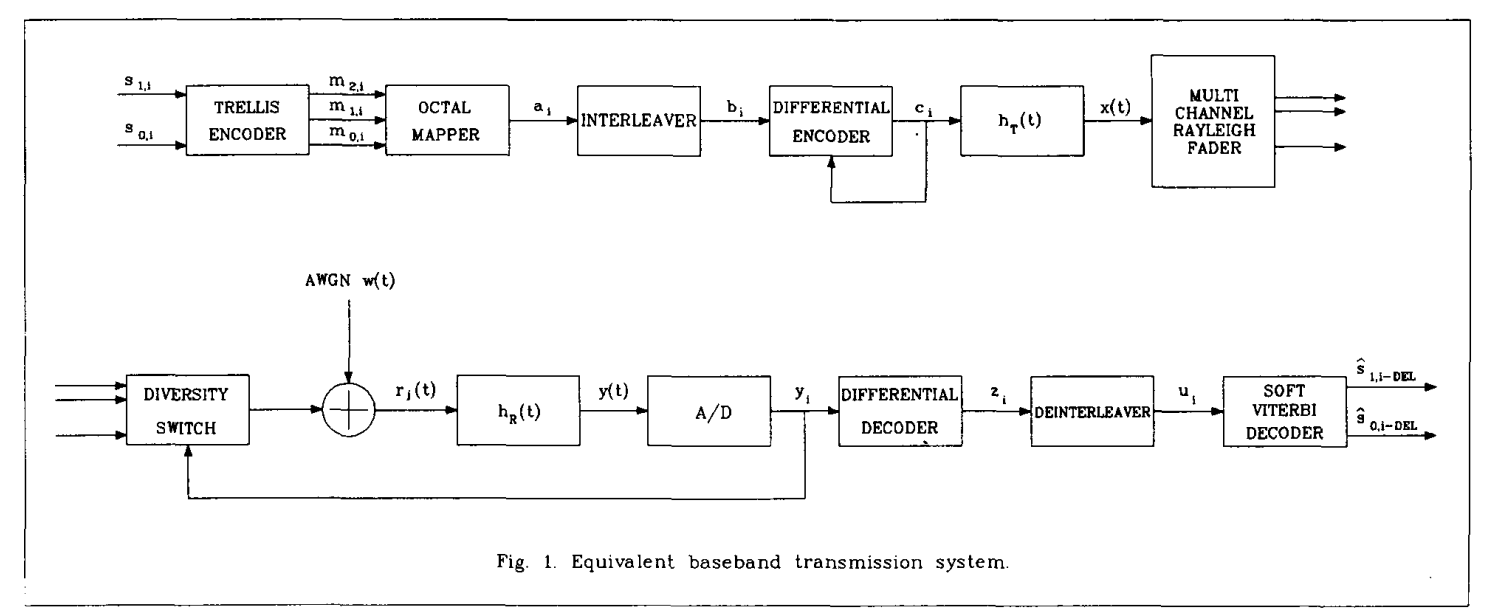

quency control (AFC) and clock recovery are assumed. For modeling the mobile radio channel we have considered a complex-valued stochastic process, $A(t)$, which describes multiplicative non-frequency selective fading. The envelope $|A(t)|$ and the phase $\operatorname{Arg}[A(t)]$ have Rayleigh and Uniform distributions, respectively. The baseband doppler power spectrum associated with this channel has been taken to be, [1],

$$
A(f)=\left\{\begin{array}{cl}
{\left[1-\left(f / f_{d}\right)^{2}\right]^{-1 / 2},} & \text { for }|f| \leq f_{d} \\
0, & \text { for }|f|>f_{d}
\end{array}\right.
$$

where $f_{d}$ is the maximum doppler shift frequency. Real and imaginary parts of the Rayleigh channel samples having this spectrum have been generated by shaping two statistically independent white Gaussian random sequences with a 6th order low pass filter.

At the emitter, a data input bit pair $s_{i}=\left(s_{0, i}, s_{1, i}\right)$ is fed into a rate $2 / 3$ trellis encoder at time iT, which generates the coded 3-bit word $m_{i}=\left(m_{0, i}, m_{1, j}, m_{2 j}\right)$ as a function of its state at the instant (i-1)T and of the input datasymbol $s_{i}$. The octal mapper, applying mapping by set partitioning [3], converts the 3-bit word $m_{i}$ into the coded 8-PSK symbol $a_{i}$ as given by equation (2),

$$
a_{i}=\exp \left\{j \cdot\left[m_{2, i} \cdot \pi+m_{1, j} \cdot \frac{\pi}{2}+m_{0, i} \cdot \frac{\pi}{4}\right]\right\}
$$

These coded symbols are fed into the block interleaver that can be regarded as a buffer, with NL rows and NC columns, that breaks the channel memory and makes full use of the Ungerboeck codes. NL and NC represent, respectively, the span and the depth of interleaving. The data symbols $\left\{a_{i}\right\}$ are stored into the buffer in successive columns and read out in rows. In [5] and [6], NL and NC were chosen such that their equivalent time spans were equal to or larger than the delay introduced by the soft Viterbi decoder and fade duration, respectively. In this paper, the effects of reducing these parameters will be shown. Differential encoding of the interleaver output symbols, $b_{i}$, yields the coded 8-DPSK symbol,

$$
c_{i}=c_{i-1} \cdot b_{i}
$$

which once filtered by the transmission filter

provides the baseband complex transmitted signal,

$$
x(t)=\sum_{k} c_{k} \cdot h_{T}(t-k T)
$$

where $h_{T}(t)$ is the impulse response of the transmission filter.

In the mobile radio channel the transmitted signal is affected by a multiplicative nonfrequency selective fading, $\mathrm{A}(\mathrm{t})$. Thus, the receiver input signal, assuming that it is connected to the $j$-th diversity antenna $(j=1,2)$, can be written in the form,

$$
r_{j}(t)=\sum_{k} c_{k} \cdot A_{j}(t) \cdot h_{T}(t-k T)+w(t)
$$

where $w(t)$ is an additive white Gaussian noise. Assuming that a switch has taken place at the instant $t=N T$,we will obtain, at the reception filter output, the complex baseband signal, 


$$
\begin{aligned}
y(t)= & {\left[\sum_{k} c_{k} \cdot A_{j-1}(t) \cdot h_{T}(t-k T) \cdot u(-t+N T)\right] * h_{R}(t)+} \\
& +\left[\sum_{k} c_{k} \cdot A_{j}(t) \cdot h_{T}(t-k T) \cdot u(t-N T)\right] * h_{R}(t)+n(t)= \\
& =\left[\sum_{k} c_{k} \cdot A_{j-1}(t) \cdot h_{T}(t-k T) \cdot u(-t+N T)\right] * h_{R}(t)+ \\
& +\left[\sum_{k} c_{k} \cdot A_{j-1}(t) \cdot h_{T}(t-k T) \cdot u(t-N T)\right] * h_{R}(t)- \\
& -\left[\sum_{k} c_{k} \cdot A_{j-1}(t) \cdot h_{T}(t-k T) \cdot u(t-N T)\right] * h_{R}(t)+ \\
& +\left[\sum_{k} c_{k} \cdot A_{j}(t) \cdot h_{T}(t-k T) \cdot u(t-N T)\right] * h_{R}(t)+n(t)
\end{aligned}
$$

where $h_{R}(t)$ is the impulse response of the reception filter, $u(t)$ represents the unit step function and $n(t)$ is an additive filtered Gaussian noise. Assuming a slow Rayleigh fading and that

$$
h_{N}(t)=\left[h_{T}(t) \cdot u(t-N T)\right] * h_{R}(t)
$$

expression (6) can be rewritten in the form,

$$
\begin{aligned}
y(t) & =\sum_{k} c_{k} \cdot A_{j-1}(t) \cdot h(t-k T)+ \\
& +\left[A_{j}(t)-A_{j-1}(t)\right] \cdot \sum_{k} c_{k} \cdot h_{N}(t-k T)+n(t)
\end{aligned}
$$

where $h(t)$ is the overall impulse response of the transmission system in absence of channel distortion. This expression describes the behavior of the switched diversity system on both transient and permanent states when only one switch is generated or when successive switches are generated once finished the transient state due to the first switch Thus, expression (8a) is a valid model for the switch and stay strategy. When working on the permanent state (8a) can be simplified as in (8b),

$$
y(t)=\sum_{k} c_{k} \cdot A_{j}(t) \cdot h(t-k T)+n(t)
$$

The received signal is sampled by an A/D converter at time $t_{i}=i T-\tau$ yielding the complex sample $y_{i}$. Assuming a perfect clock recovery, $\tau=0$, we have $h(0)=1$ and $h((i-k) T)=0$, for all $k \neq i$, and so

$$
y_{i}=c_{i} \cdot A_{j-1, i}+\left[A_{j, i}-A_{j-1, i}\right] \cdot \sum_{k} c_{k} \cdot h_{N}(i T-k T)+n_{i}
$$

where $A_{j, i}=A_{j}(i T)$. When working on the permanent state (9a) can be simplified as in (9b),

$$
y_{i}=c_{i} \cdot A_{j, i}+n_{i}
$$

For the Nyquist system one can assume the noise samples $n_{i}$ to be uncorrelated and so, the average bit energy to spectral noise power density ratio is, for $\tau=0$,

$$
\frac{E_{b}}{N_{0}}=\frac{E\left\{\left|A_{j, i}\right|^{2}\right\} \cdot E\left(\left|c_{i}\right|^{2}\right\}}{2 \cdot \sigma^{2}}
$$

where $\mathrm{E}\{\cdot\}$ denotes expectation and $\sigma^{2}$ is the variance of the thermal noise in each dimension.

Symbol detection is achieved by a differential decoder which uses the received symbol at the instant (i-1)T as phase reference, so, the detected symbol can be written as

$$
z_{i}=y_{i} \cdot y_{i-1}^{*}=c_{i} \cdot c_{i-1}^{*} \cdot A_{j, i} \cdot A_{j, i-1}^{*}+v_{i}=d_{i}+v_{i}
$$

where $v_{i}$ is a complex noise sample which, due to the multiplication, is non-Gaussian and correlated. Once performed the differential decoding, the detected samples are stored into the block deinterleaver in rows and read out in columns for soft Viterbi decoding.

The trellis encoder used at the transmitter side works as a "finite state machine", then the Viterbi decoder will be optimum for estimating the maximum likelihood coded symbol sequence. But the noise samples at the Viterbi decoder input are nonGaussian and correlated. Thus, the use of a proper metric is needed. The cumulative metric that we have used for the path ending at state $\mu$ at time iT is given by the negative increase of the quadratic Euclidean distance over a symbol period [6],

$$
Q_{\mu, i}=\sum_{k=0}^{M}\left|z_{i-k}-A_{j, j-k} \cdot A_{j, i-k-1}^{*} \cdot \hat{d}_{k, i-k}\right|^{2}
$$

where $M$ is the soft Viterbi decoding delay. It has been assumed that two consecutive samples of the Rayleigh mobile channel are approximately equal (slow Rayleigh fading),

$$
A_{j, i-k} \cong A_{j, i-k-1}
$$

this assumption applies for transmitter data rates much greater than the doppler frequency of the mobile channel.

\section{COMPUTER SIMULATION RESULTS}

In this Section a computer simulation procedure is carried out in order to assess the performance of the system presented in Section II. We simulate the uncoded 4DPSK and coded TCM-8DPSK mo- 


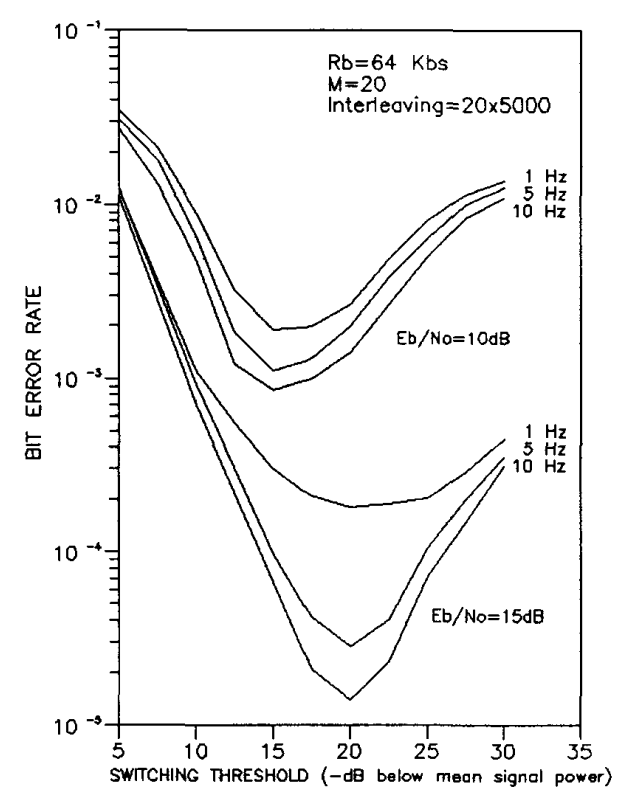

Fig. 2. Relation between BER and switching threshold with $\mathrm{Eb} /$ No and doppler frequency as parameters.

dulation schemes, with and without switched diversity. The results obtained for 4DPSK modulation scheme are considered as a reference to determine the combined coding and diversity gains. We investigate the dependence of BER on signal to noise ratio, switching threshold, doppler frequency and interleaving buffer size. In particular we determine the relation between BER and switching threshold with all other variables as parameters. From these results, we determine the optimum switching threshold as a function of these parameters. Using this optimum threshold we find the performance of 4DPSK and TCM-8DPSK modulation schemes with switch and stay-strategy. In all the cases we consider a non-selective mobile radio channel, a bit rate of $64 \mathrm{Kbits} / \mathrm{s}$, a 20 symbol path memory in the Viterbi decoder, doppler frequencies ranging from $1 \mathrm{~Hz}$ to $50 \mathrm{~Hz}$ and different interleaving buffer sizes. Typically, at least 500 symbol errors are associated with each data point.

Fig. 2 shows the relation between switching threshold, in $\mathrm{dB}$ below the mean signal level, and BER for a doppler frequency of 1,5 and $10 \mathrm{~Hz}$ and $E_{b} / N_{0}$ values of 10 and $15 \mathrm{~dB}$. These results are for an interleaving buffer size of $20 \times 5000$ symbols. For a given doppler frequency and signal to noise ratio there exists an optimum switching threshold that minimizes the BER. This minimum comes from a trade off between switching-produced and noise-produced errors. Due to the independence between instantaneous phases of the received signals a switch produces a phase transient that implies a high error probability for a symbol received at this time. As the switching threshold increases toward higher

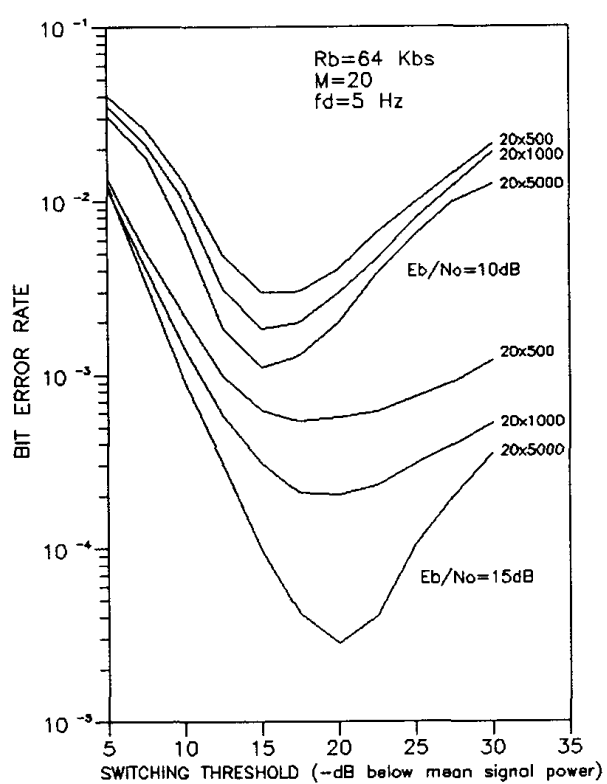

Fig. 3a. Relation between BER and switching threshold with $\mathrm{Eb} /$ No and depth of interleaving as parameters.

relative levels, the number of threshold crossings of the signal, and thus the number of switches, increases. Then, the greater the switching threshold the greater the number of switching-produced errors. As the switching threshold decreases toward lower relative levels, the instantaneous signal to noise ratio preceding a switch decreases also and then a greater number of noise-produced errors is generated. Thus, the lower the switching threshold the greater the number of noise-produced errors. For a fixed switching threshold and a given doppler frequency the number of threshold crossings of the signal and thus switching produced errors are relatively unaffected by the signal to noise ratio Therefore, as the signal to noise ratio increases and the number of noise-produced errors decreases the optimum threshold moves toward lower signal values. Fig. 2 also shows that doppler frequency has little effect on the optimum switching threshold.

Figs. $3 a$ and $3 b$ show the effect of interleaving buffer size on the relation between switching threshold and BER. Results are shown for a doppler frequency of $5 \mathrm{~Hz}$. As we can see neither depth nor span of interleaving have a remarkable effect on the location of the optimum switching threshold. For a span of interleaving of 20 symbols and an $E_{b} / N_{o}=10 \mathrm{~dB}$ the optimum threshold remains close to $15 \mathrm{~dB}$ for depths of interleaving ranging from 500 to 5000 symbols. At an $\mathrm{E}_{\mathrm{b}} / \mathrm{N}_{0}=15 \mathrm{~dB}$ the optimum threshold increases from $17.5 \mathrm{~dB}$ at a depth of interleaving of 500 symbols to $20 \mathrm{~dB}$ at a depth of interleaving of 5000 symbols. For a depth of inter- 


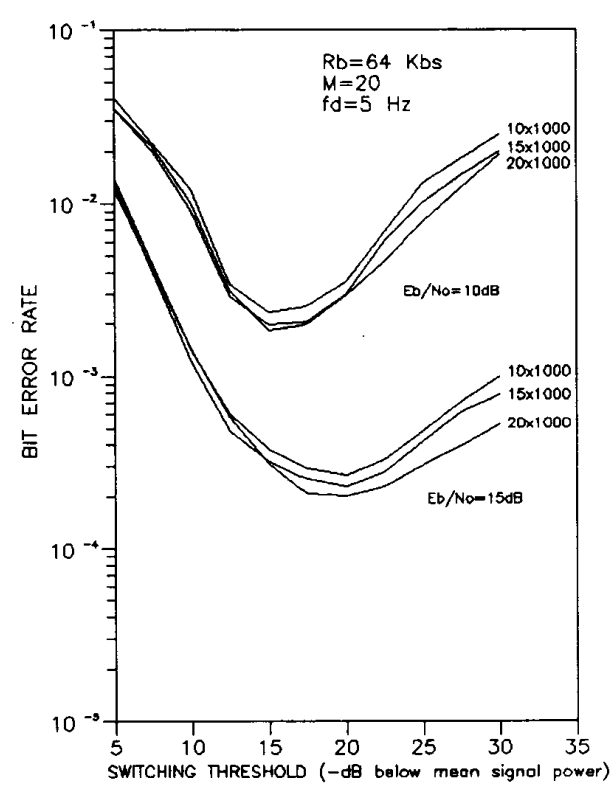

Fig. 3b. Relation between BER and switching threshold with $\mathrm{Eb} / \mathrm{No}$ and span of interleaving as parameters.

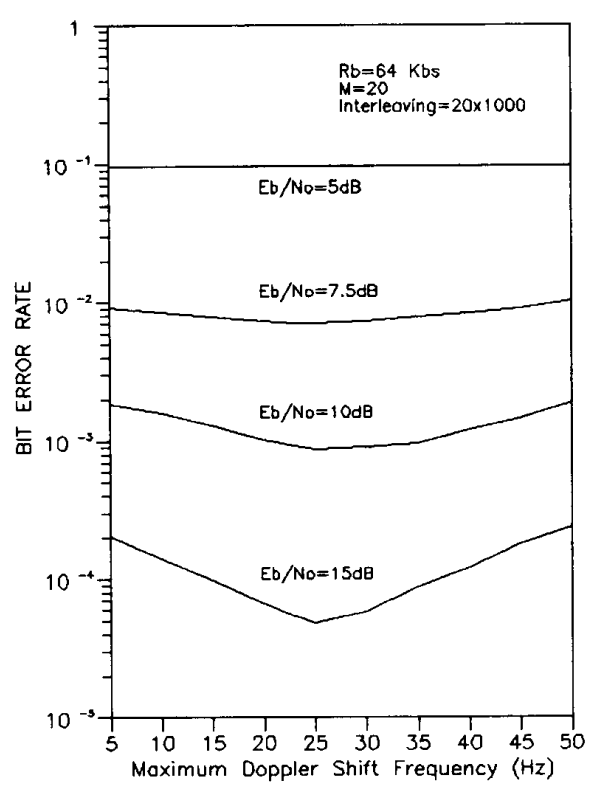

Fig. 4. BER-Performance of TCM-8DPSK for frequency as parameter. leaving of 1000 symbols and spans of interleaving ranging from 10 to 20 symbols the optimum threshold remains close to $15 \mathrm{~dB}$ for an $\mathrm{E}_{\mathrm{b}} / \mathrm{N}_{0}=10 \mathrm{~dB}$ and close to $20 \mathrm{~dB}$ for an $\mathrm{E}_{\mathrm{b}} / \mathrm{N}_{0}=15 \mathrm{~dB}$.

Figs. 2 and 3 show that the location of the optimum switching threshold is nearly constant for different interleaving buffer sizes and practically unaffected by the doppler frequency. Thus, the knowledge of the mean signal level allows the implementation of the switching strategy using an IF AGC amplifier and a fixed level detector [7].

The performance of switched-diversity TCM8DPSK system with optimum threshold is shown in Figs. 4, 5a and 5b. Fig. 4 shows the BER as a function of doppler frequency for $E_{b} / N_{0}$ ratios ranging from 5 to $15 \mathrm{~dB}$ and an interleaving buffer size of $20 \times 1000$ symbols. As the doppler frequency increases the average fade duration decreases proportionally and the effects of interleaving become more prominent. But the average number of threshold crossings, and thus the number of switching-produced errors, increases proportionally to doppler frequency. Then, for a given interleaving buffer size there exists a doppler frequency that minimizes the BER. This minimum arises from a trade off between efficiency of the interleaving process and switching-produced errors. Figs. 5a and $5 b$ show the BER as a function of depth and span of interleaving respectively, for a doppler frequency of $5 \mathrm{~Hz}$ and $\mathrm{E}_{\mathrm{b}} / \mathrm{N}_{0}$ ratios ranging from 0 to $15 \mathrm{~dB}$. Lowering the interleaving buffer size a BER degradation arises.
However, only $1.5 \mathrm{~dB}$ of degradation appear when it is reduced from $20 \times 5000$ symbols to $10 \times 1000$ symbols, which could make the delay time suitable for a great deal of applications.

Finally, in order to establish a comparison between different receiver strategies, Fig. 6 shows the performance of 4DPSK, switched-diversity 4DPSK, TCM-8DPSK and switched-diversity TCM-8DPSK systems. Results are shown for a bit rate of $64 \mathrm{Kbits} / \mathrm{s}$, a doppler frequency of $10 \mathrm{~Hz}$ and an interleaving buffer size of $20 \times 5000$ symbols. It can be noted that, with these parameters, the switched-diversity TCM-8DPSK system improves the performance of all the other systems. In particular, it improves the switched-diversity 4DPSK performance in more than $8 \mathrm{~dB}$ for a $\mathrm{BER} \leq 10^{-3}$.

\section{CONCLUSIONS}

In this paper we have analized the performance of 4DPSK and TCM-8DPSK systems, with and without switched diversity, on the Rayleigh fading channel. BER has been obtained as a function of switching threshold, doppler frequency and block interleaving buffer size. In particular we have determined the optimum threshold as a function of the other parameters. We have found that the location of the optimum switching threshold is nearly constant for different interleaving buffer sizes and practically unaffected by the doppler frequency. Using the optimum switching threshold we have determined the BER-performance of the systems with doppler frequencies and interleaving buffer size as parameters. One of the most significant 


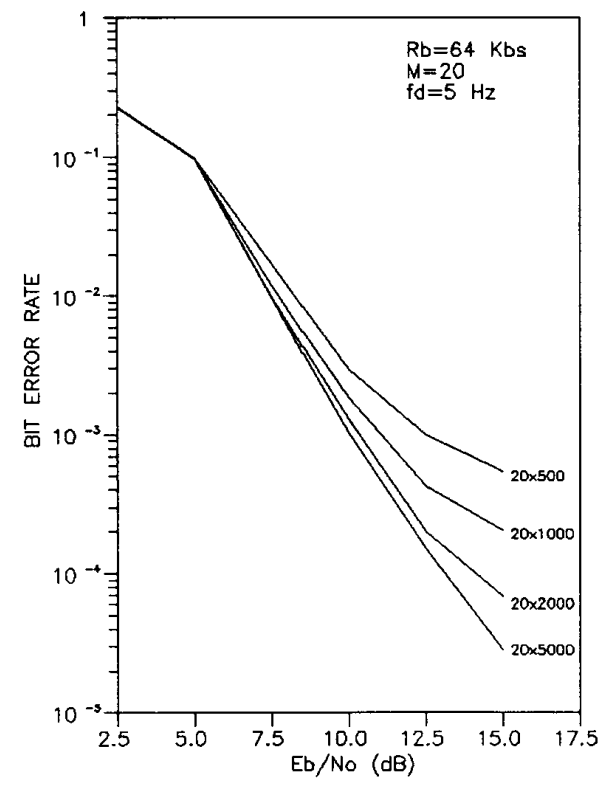

Fig. 5a. BER-Performance of TCM-ADPSK for wo-branch switched diversity with depth of interleaving as parameter.

results is that the switched-diversity TCM-8DPSK system improves the switched-diversity 4DPSK performance in more than $8 \mathrm{~dB}$ for a BER $\leq 10^{-3}$, a doppler frequency of $5 \mathrm{~Hz}$ and an interleaving buffer size of $20 \times 5000$ symbols. By increasing this figure to $10 \times 1000$ the improvement is only reduced by $1.5 \mathrm{~dB}$.

\section{ACKNOWLEDGMENT}

This work was supported by CICYT (Spain) under Grant TIC 88053

\section{REFERENCES}

|1 $\mid$ JAKES, W.C., Jr., Microwave Mobile Communications, John Wiley, New York, 1974.

$|2|$ JUSTAKO, A.J.,Jr., "Performance of Feedback and Switch Space Diversity $900 \mathrm{MHz}$ FM Mobile Radio Systems with Rayleigh Fading", IEEE Trans. on Vehic. Tech., vol VT-22, Nov. 1973.

|3| UNGERBOECK, G., "Channel Coding with Multilevel/Phase Signals", IEEE Trans. on Inf. Theory, vol IT-28, Jamuary 1982.

|4| FORNEY, G.D. et alt., "Efficient Modulation for Band-Limited Channels", IEEE Jour. on Sel. Areas in Corm., Sep.1984.

|5| DIVSALAR, D. et alt., "Trellis Coded Moculation for 4800$9600 \mathrm{bits} / \mathrm{s}$ Transmission Over a Fading Mobile Satellite Channel", IEEE Jour. on Sel. Areas in Corm., Feb. 1987.

|6| EDBAUER, F., "Coded 8-DPSK Modulation with Differentially Coherent Detection. An efficient Modulation Scheme for Fading Channels", GLOBвCOM'87.

$|7|$ AFNOLD, H.W. et alt., "Switched- Diversity FSK in Frequency Selective Rayleigh Fading", IFFE Trans. on Vehic. Tech. vol VT-33, August 1984.

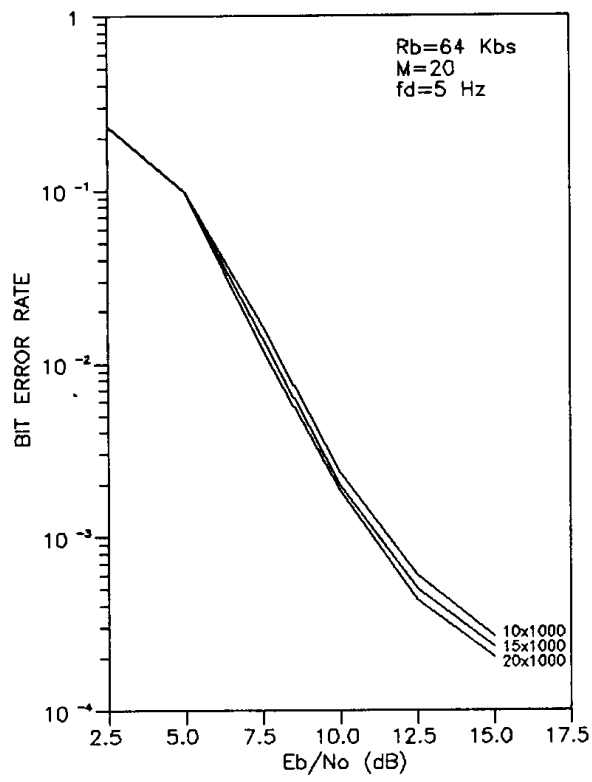

Fig. 5b. BER-Pertormance of TCM-BDPSK for tro-branch switched diversity with span of interleaving as parameter.

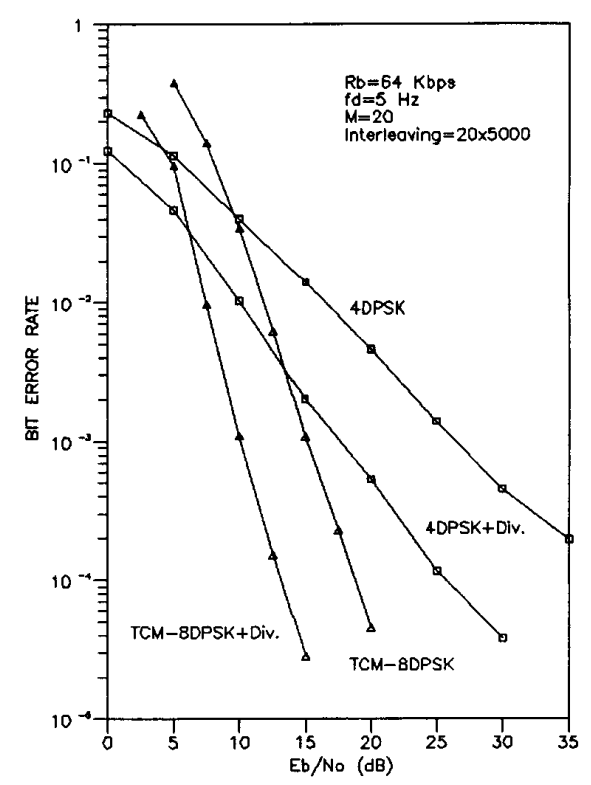

Fig.6. BER-Performance of Uncoded 4DPSK and TCX-8DPSK for a Rayleigh channel, wth and without switched-diversity. 